\title{
RADIATION PATTERN OPTIMIZATION BY LOADED IMPEDANCE IN THE BASE OF PASSIVE ELEMENT
}

\author{
Marek Dvorský *
}

\begin{abstract}
This article deals with optimizing the radiation pattern by using two vertical middle wave antenna arrays. Optimization is achieved by adding an appropriate impedance into the base of the passive antenna radiator. The proposed solution deals with finding the best value of the loaded impedance. The final result is to achieve the most effective shape of the radiation pattern. The proposed method was finally approved by measurement on a real middle wave antenna array.

K e y w or d s: antenna, radio pattern, load impedance, DRM, optimization
\end{abstract}

\section{INTRODUCTION}

The article deals with an upgrade of the existing middle wave antenna array to antennas suitable for digital radio broadcasting systems. The first part of the article tries to explain the reasons of our choice to deal only with the two-element antenna array. The solution of this issue is connected to the starting digitalization process at radio bands bellowing to $30 \mathrm{MHz}$. The following part provides essential mathematical tools necessary to derive the radiation pattern and to construct the proposed mathematical model.

The inputs into the proposed mathematical model are the geometric dimensions of radiators and their lay-out. The output value is loaded impedance in the base of passive element, which forms the radiation characteristics regarding to the antenna array operator requirements.

Verification of mathematical model was carried out using the development environment of software Mathcad. In this program were implemented mathematical relationships and calculated the value of inserted impedance. The shape of the radiation was then verified with the simulation program EZNEC v5.0.20. Finally, the proposed model was verified by measurement on the real middle wave antenna array.

\section{THE IMPORTANCE OF THE EXISTING MW BROADCASTING CENTERS}

Frequency positions on Middle Waves (MW) are firmly committed to the international notification of the geographical area according to the registered geographical coordination, for a maximum radiated power (EIRP) and the particular time interval. Notifications are entered in Master International Frequency Register (MIFR), which is conducted in the International Telecommunication
Union Radiocommunication Bureau (ITU-BR), in the frequency plan GE75. The national frequency allocation for The Czech Republic includes the MW band frequencies for the 24-hour operation, as well as frequencies to operate daily between 4 to 17 o'clock GMT. MW frequency allocation includes The Czech Republic only 4 high quality with low levels of frequencies night interference from foreign transmitters: 639, 954, 1233 and $1287 \mathrm{kHz}$. These frequencies allow creating networks with a nationwide coverage. The national frequency allocation includes an additional frequency $1071 \mathrm{kHz}$ with more night disturbance. Further frequency positions, notified only for daily operations are: 846, 864, 900, 1017 and $1593 \mathrm{kHz}$.

Under the above given circumstances, only four 24 hour frequencies are recorded in certain positions of existing transmitters. These could be possibly used to create an Amplitude Modulated (AM) broadcast in 2 national transmitters' networks. To take advantage of the digital broadcasting system Digital Radio Mondiale (DRM) [4] it could be possible to create in the Czech Republic 4 programs in nationwide Single Frequency Networks (SFN). There is also a spare antenna array at Litomyšl-Pohodlí recorded the frequency $1287 \mathrm{kHz}$, where a verification measurement was made to confirm the theoretical conclusions of the proposed mathematical model.

The current antenna systems meet the requirements of classical analogue broadcasting, but with the advent of digitization it will be necessary to assess whether to build new antenna systems or to use the existing ones, which is certainly not going to be without their adjustments.

In this context, there is also offered a question of optimization of the radiation pattern. Antenna systems which have all the elements actively fed can often be usefully adapted to a system with a passive tuned element. This will not only simplify the power supply antenna system but also the flexibility of any changes in the radiation pat-

\footnotetext{
* Department of Telecommunications, FEECS VŠB — Technical University Ostrava, 17. listopadu 15, 708 33 Ostrava-Poruba, Czech republic, marek.dvorsky@vsb.cz
} 


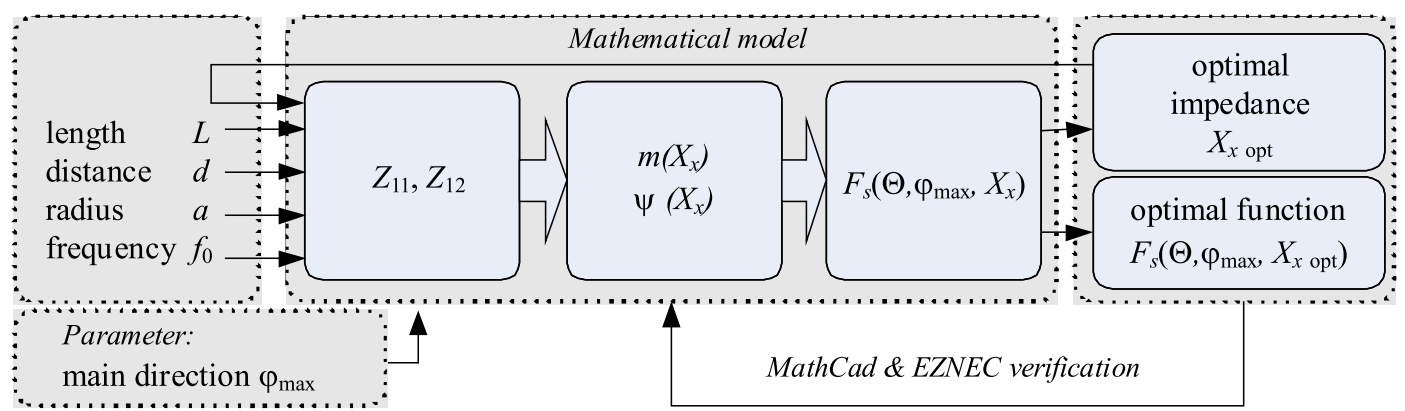

Fig. 1. Mathematical model proposed optimization

tern. The following section contains the proposed mathematical model.

\section{PROPOSED MATHEMATICAL MODEL}

The proposed mathematical model (Fig. 1) describes the optimization of the radiation pattern of the two vertical radiators. Only one of them is actively powered.

The reasons of the choice of this type of antenna array are related to the best simplicity and efficiency of this type of antenna lay-out. The mathematical model describes how to find an effective value load impedance $X_{x}$ to achieve the maximum value of the radiation function $F s\left(\theta, \varphi, X_{x}\right)$ in the required direction of the maximum $\varphi_{\max }$. The basic precondition for this kind of antenna system are two geometrically identical linear vertical radiators with a sine current distribution. The antenna towers are placed on a base insulator through which one antenna is fed. The second tower is grounded via a loaded impedance $X_{x}$ to the Earth's surface, assumed to be ideally conductive, forming a mirror reflection of the antenna system.

The inputs into the proposed mathematical model (Fig. 1) are geometrical proportions of two equal antenna towers: Length of tower $L(\mathrm{~m})$; Spacing $d(\mathrm{~m})$; Radius of towers $a(\mathrm{~m})$ and working frequency $f_{0}(\mathrm{~Hz})$. The main parameter for optimization is the direction of the main lobe $\varphi_{\max }$. The outputs are at the first step the optimal value of the loaded impedance $X_{\mathrm{x}_{\text {_opt }}}$. This value is then inserted into the radiation pattern calculation to achieve the main aim, which is the optimal shape of the radiation pattern (3).

The proposed mathematical model is based on calculating the optimal shape of the radiation pattern $F s\left(\theta, \varphi, X_{x}\right)$. It is based on the known self and mutual impedances $Z_{11}(L)=R_{11}+X_{11}$ and $Z_{12}(d)=R_{12}+X_{12}$ [4]. The next step consists in the calculation of the following current ratio in the antenna towers $m\left(X_{x}\right)(1)$ and the phase $\psi\left(X_{x}\right)(2)$ depending on $X_{x}$.

\subsection{Radiation pattern}

The derivation of the radiation functions $F s\left(\theta, \varphi, X_{x}\right)$ for the array with a passive element is necessary to know the circumstances of the amplitude ratio (1) and the phase (2) currents of single emitters. The size and phase of current in the passive element depends on the electrical properties and the reciprocal arrangement in relation to the actively fed element antenna.

Then the phase and the amplitude ratio of the size of current in antenna elements that are functions of the values of the embedded impedance $X_{x}$ can be expressed $[2]$

$$
\begin{aligned}
& m\left(X_{x}\right)=\sqrt{\frac{R_{12}^{2}+X_{12}^{2}}{R_{11}^{2}+\left(X_{11} \pm X_{x}\right)^{2}}}, \\
& \psi\left(X_{x}\right)=\pi+\operatorname{arctg} \frac{X_{12}}{R_{12}}-\operatorname{arctg} \frac{X_{11} \pm X_{x}}{R_{11}} .
\end{aligned}
$$

The radiation pattern can be drawn in the plane $E(\varphi=$ $0)$, and the plane $H(\theta=\pi / 2)$ as the absolute value of radiation group functions

$$
F s\left(\theta, \varphi, X_{x}\right)=F(\theta, \varphi)\left|K\left(\theta, \varphi, X_{x}\right)\right|
$$

where:

$$
\begin{array}{r}
K\left(\theta, \varphi, X_{x}\right)=\left|1+m\left(X_{x}\right) e^{j \psi\left(X_{x}\right)} e^{j(k d \sin \theta \cos \varphi)}\right|= \\
\sqrt{1+m\left(X_{x}\right)^{2}+2 m\left(X_{x}\right)\left(k d \sin \theta \cos \varphi+\psi\left(X_{x}\right)\right)},
\end{array}
$$

$K\left(\theta, \varphi, X_{x}\right)$ is an interference function.

\subsection{Optimal value inserted impedance}

In a depth analysis it can be assumed that if the closest form of the lobe in the plane $E F s\left(\theta, 0, X_{x}\right)$ is desirable, most energy should be radiated at the angle $\varphi \rightarrow \pi / 2$. The aim is therefore to find that embedded impedance $X_{x}$ value which reaches the greatest possible value of the maximum function (the size of the lobe) $F s\left(\theta, 0, X_{x}\right)$. Optimizing the shape of the radiation pattern for two vertical radiators is therefore based on the search for the maximum radiation function $F s\left(\theta, 0, X_{x}\right)(3)$. 


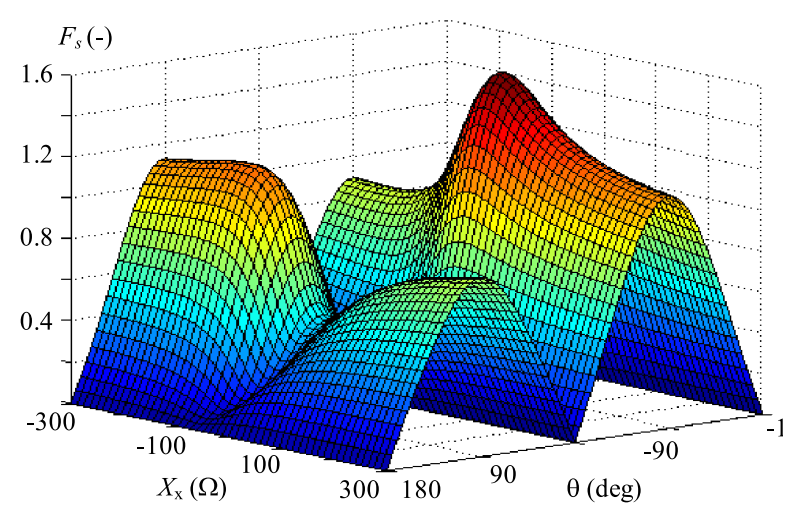

Fig. 2. 3D Radiation pattern of two unipol Antennas with load impedance $X_{x}$ in $2 \mathrm{D}$ plot + parameter
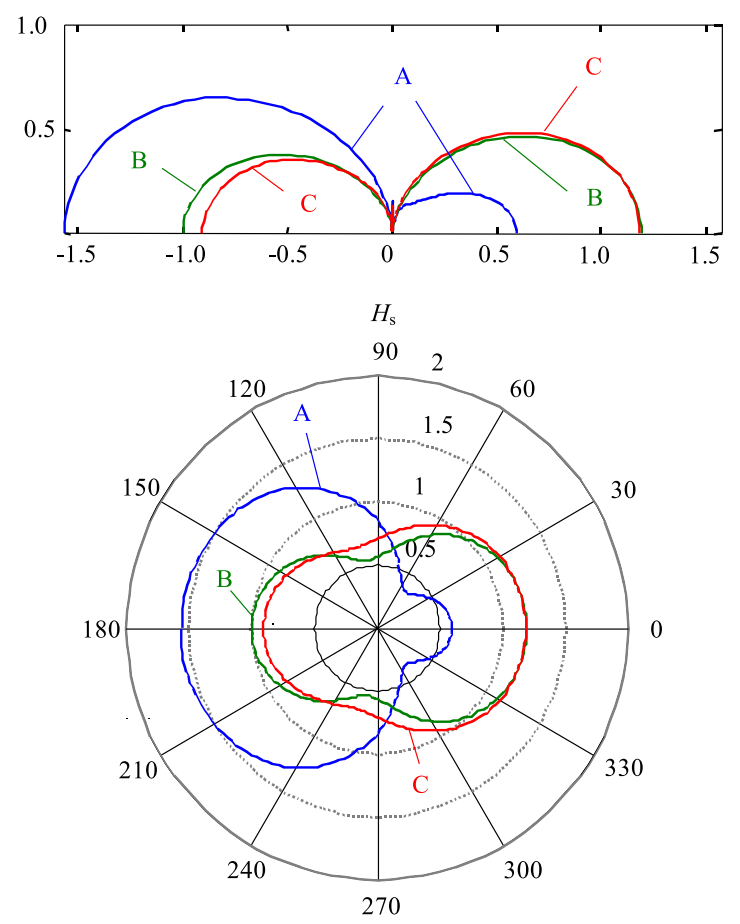

Fig. 3. Vertical pattern $F s\left(\theta, 0, X_{x}\right)$ (up); Horizontal pattern $F s\left(\pi / 2, \varphi, X_{x}\right)$ (below)

Previously the derived relations (1)-(4) were implemented into the development environment software Mathcad from Mathsoft Engineering \& Education, with the added library for Visual Electromagnetic Mathcad [3]. In addition, by the above-mentioned software tools the positions of the absolute maximum functions $F s\left(\pi / 2, \varphi_{\max }, X_{x}\right)$ shown in Fig. 3 were found. The main aim is to find such a point where the value of the derivative of function $F s\left(\theta, \varphi_{\max }, X_{x}\right)$ with respect to $\mathrm{Xx}$ is zero

$$
\frac{\partial F s\left(\frac{\pi}{2}, 0, X_{x}\right)}{\partial X_{x}}=0
$$

The main requirement is to find the minimum backward factor for the reflector type of antenna system, so the value of $\varphi=(2 n-1) \pi, n=1,2,3, \ldots$

$$
\frac{\partial F s\left(\frac{\pi}{2}, \pm(2 n-1) \pi, X_{x}\right)}{\partial X_{x}}=0 ; n=1,2,3, \ldots
$$

Selecting the appropriate angle $\theta=\pi / 2$ defines the vertical plane $E, \varphi_{\max }=0$ and determines the direction of the lobe in the direction of the axis of the antenna system. After finding the absolute maximum of the function $F s\left(\theta, \varphi_{\max }, X_{x}\right)$ it is necessary to determine the appropriate value of $X_{x}$ which corresponds to the found maximum (Fig. 2).

\section{EVALUATION}

The final step for the good verification of the achieved results accuracy can be performed with drawing the horizontal and vertical radiation characteristics in the spherical coordinate system with the optimized value loaded impedance $X_{\text {x_opt }}$. By comparing the characteristics of the different loaded impedance values, it is obvious that the value of $X_{\mathrm{x} \_o p t}$ corresponds to the optimal/maximal shape of the radiation pattern (Fig. 3, up and Fig. 3, down).

Curve A (blue) in Fig. 3, describes the antenna system with the loaded impedance with optimum value $X_{\mathrm{x}_{\mathrm{o} o p t}}$, curve $\mathrm{C}$ (red) represents the optimization for the redirection and curve $\mathrm{B}$ (green) shows the general value of the loaded impedance. It can be clearly seen that $\mathrm{A}$ diagram (blue) reaches the best values of $F s\left(\theta, \varphi_{\max }, X_{x}\right)$ in the direction $\varphi_{\max }=180^{\circ}$ (analyzed direction). This approves the theory of the proposed mathematical model.

\section{CONCLUSION}

The presented article has drawn out the optimization of the radiation pattern in a middle wave antenna array in the context of the potential use of the existing antenna systems for digital radio signal distribution such as DRM. This study is focused on middle wave vertical antenna systems that appear to be the most prospective for digital broadcasting below $30 \mathrm{MHz}$ (reasons are listed in Chapter 2).

Nowadays, optimization of the radiation pattern for the existing antenna systems is also an issue for the context of digital radio broadcasting expansion. Antenna systems which have all the elements actively fed can be often usefully adapted to a system with a passive tuned element. This will not only simplify the power supply for the antenna systems but also gives flexibility for any changes in antenna radiation pattern.

The presented model is currently unique which deals with the issues of re-using the existing middle wave antenna systems. The benefit for this practice is creating a new procedure to calculate technically the optimized radiation antenna pattern of a two linear element antenna array with a passive element inserted in the base of 
the passive element. The proposed mathematical model is based on mathematical calculations of integral \& differential equations which are supplemented by new findings.

Under the given circumstances, a "Radiation diagrams dictionary" could be created for the most widely used middle waves antenna configurations.

\section{REFERENCES}

[1] DVORSKY, M.: Optimization of Radiation Pattern by Loaded Impedance in the Base of Passive Element, PhD thesis, VSB Technical University of Ostrava, 2008.

[2] CAHA, V.-PROCHAZKA, M.: Antennas (Antńy), SNTL press, Praha, 1956. (in Czech)

[3] Mathcad (online). Avaliable from http://www.ptc.com, accessed 2008-06-13.

[4] VAVRA, S.-TURAN. J.: Antennas and Electromagnetic Waves Propagation (Antény a šírenie elektromagnetických vín), ALFA, Bratislava, 1989. (in Slovak)
[5] DVORSKY, M.: Digital Radio in the Broadcasting Bands bellow $30 \mathrm{MHz}$, In 7. Seminar of Telecommunications Department, Ostrava, 2007.

[6] BALANIS, C. A.: Antenna Theory: Analysis and Design, 3rd edition, United States, 2005.

[7] KING, R. W.: The Theory of Linear Antennas, Cambridge, 1956.

Received 12 March 2010

Marek Dvorský (Ing, PhD), born in 1981, is a researcher at the Faculty of Electrical Engineering and Computer Science of VSB Technical University of Ostrava, Czech Republic. He graduated from the FEECS of the same university with thesis topic Middle wave antenna duplexer in 2004. Recently he received a PhD as he worked on Optimization of Radiation Pattern by Loaded Impedance in the Base of Passive Element'. Currently, he works as an assistant professor at the Department of Telecommunications, FEECS VSB TU Ostrava. The main fields of his professional interest include modern digital broadcasting systems such as DVB, DAB, DRM etc. 